\title{
Une mémoire apaisée ? \\ La demande de reconnaissance du génocide des Arméniens dans l'espace public québécois 1995-2015
}

En 1995, la communauté arménienne du Québec offrait à la Ville de Montréal un monument intitulé La Réparation pour commémorer les victimes de génocides au $X X^{e}$ siècle, $y$ compris le génocide des Arméniens durant la Première Guerre

L'auteure : Jocelyne СНАВOT est Professeure agrégée au Département d'histoire et de géographie de l'Université de Moncton, Canada. mondiale. Pendant trois ans, le débat opposera les partisans et les opposants à la commémoration des victimes de cet événement. À l'époque, l'affaire fera grand bruit dans l'espace public médiatique québécois. Ainsi, de nombreuses questions surgiront quant à la pertinence de commémorer un génocide non reconnu par le Canada, par une partie de la communauté internationale, de même que par l'État turc ? Ou encore, sur l'opportunité d'ériger un monument aux victimes de génocides pour la société québécoise contemporaine? Ces défenseurs estimeront que devant le déni des autorités turques, la principale arme de la communauté arménienne demeure l'expression culturelle et mémorielle de son identité intimement liée au génocide de 1915. L'érection d'un monument commémoratif dans l'espace public devient ainsi l'expression tangible de la mémoire partagée du génocide des Arméniens par l'ensemble des collectivités québécoise et canadienne. Quant aux opposants, ils considèreront que la société d'accueil n'a pas à prendre position dans une controverse historique sans rapport avec l'histoire canadienne et québécoise'.

Depuis, d'autres monuments ont été réalisés à la mémoire des victimes du génocide des Arméniens, parmi ceux-ci on peut signaler le monument L'Espoir inauguré en 2013 à Laval, la troisième plus grande ville du Québec. Quant à la communauté arménienne québécoise, depuis vingt ans, elle a continué à se mobiliser et à manifester à la mémoire des victimes et en faveur de la reconnaissance du génocide de 1915-1916. Alors que l'année 2015 a marqué au Canada comme ail-

\footnotetext{
' CHAвот Joceline, "Débats et controverses au sujet d'un monument commémoratif : La Réparation (Montréal 1995-1998) ", Questions de communication, série Actes 5, Qualifier les lieux de détention et de massacre, Nancy, 2008, pp. 303-315. HeRnANDEZ Analays Alvarez, La commande publique dans un contexte de diversité ethnoculturelle : débat entourant l'érection du monument La Réparation, 1994-1998, mémoire de maîtrise en études des arts, Université du Québec à Montréal, 2010, 185 p.
} 
leurs dans le monde la commémoration du 100e anniversaire du génocide des Arméniens, qu'en est-il des débats et des controverses qui ont traversé l'espace public médiatique au cours des années 1990 ? Peut-on parler d'une mémoire apaisée au regard des nombreuses difficultés à faire reconnaître le crime commis il y a un siècle contre les Arméniens ${ }^{2}$ ?

Ces questions s'inscrivent dans un débat plus large sur les usages publics du passé dans leurs dimensions politiques et éthiques. Il ne saurait être question ici de reprendre les termes de ce débat mais rappelons que les thèmes de la mémoire, de la reconnaissance, de l'oubli et de la réparation en lien avec des événements souvent traumatiques du passé ont pris beaucoup d'importance dans nos sociétés et sont fortement médiatisés ${ }^{3}$. Le Canada et le Québec n'échappent évidemment pas à ce débat. Les pratiques symboliques, rhétoriques et commémoratives qui font référence aux représentations du passé participent pleinement des préoccupations et des enjeux actuels. Les questions et les polémiques autour de la reconnaissance des droits des Autochtones et plus particulièrement des pensionnats autochtones en sont de bons exemples ${ }^{4}$.

Notre étude examine le cas particulier des représentations mémorielles du génocide des Arméniens dans l'espace public québécois. Comme l'a souligné Lucette VALENSI, la question du génocide des Arméniens offre un cas limite d'usage politique du passé dans la mesure où coexistent deux discours incompatibles, celui qui reconnaît le génocide de 1915-1916 et celui qui le nie ${ }^{5}$. Dans le contexte canadien et québécois, nous avons déjà analysé la difficile gestion des mémoires

\footnotetext{
2 II faut souligner que malgré le déni dont il est toujours l'objet de la part des autorités turques, le caractère génocidaire des événements de 1915-1916 est reconnu par la majorité des spécialistes des phénomènes génocidaires.

${ }^{3}$ Parmi une abondante bibliographie sur le sujet nous retenons: Crivello Maryline, GARCIA Patrick, OfFEnSTADT Nicolas (dir.), Concurrences des passés. Usages politiques du passé dans la France contemporaine, Publications de l'Université de Provence, 2006, 298 p. Hartog François, Revel Jacques (dir.), Les Usages politiques du passé, Paris, Éditions de l'EHESS, 2001, 206 p. HutTon Patrick H., " Memories of Trauma : problems of Interpretation ", History and Theory, 43, 2004, pp. 249-259. LÉTOURNEAU Jocelyn, JEWSIEWICKI Bogumil, «Politique de la mémoire », Politique et Sociétés, 22, 2, 2003, pp. 3-15.

${ }^{4}$ BEAULIEU Alain, "Les pièges de la judiciarisation de l'histoire autochtone ", Revue d'Histoire de l'Amérique française, 53, 4, 2000, pp. 541-551. BRAnt Castellano Marlène, ArCHIBAld Linda, Degagné Mike (dir.), De la vérité à la réconciliation. Transformer l'héritage des pensionnats, Ottawa, Collection recherche de la Fondation autochtone de guérison, $2008519 \mathrm{p}$.

${ }^{5}$ VALENSI Lucette, «Notes sur deux histoires discordantes. Le cas des Arméniens pendant la Première Guerre mondiale », in HARTOG, François, ReVEL, Jacques (dir.), Les Usages politiques du passé, op. cit., pp. 157-168.
} 
communautaires turques et arméniennes hostiles et exclusives ${ }^{6}$. Dans cet article, nous prolongeons cette étude des pratiques et du discours mémoriels dans un contexte de diaspora, et ce, afin de saisir leur évolution, leur inscription et leur représentation dans l'espace public de la société québécoise de 1995 à 2015 à partir de deux séries d'événements qui ont retenu - quoique inégalement - l'attention des médias : l'inauguration du monument L'Espoir à Laval en 2013 et la commémoration durant l'année 2015 du $100^{\circ}$ anniversaire du génocide de 1915. Pour ce faire, nous avons constitué un corpus composé comme suit:

1- Tous les articles parus entre janvier et mai 2015 dans deux journaux nationaux, Le Devoir et La Presse soit 32 articles (nouvelles, chroniques, analyses, lettres d'opinion).

2- Des articles tirés du site web de Radio-Canada, radio diffuseur officiel canadien, ainsi que de journaux locaux et régionaux soit treize articles.

3- Des articles et des commentaires parus sur deux sites web liés à des journaux édités par la communauté arménienne: Nouvelles d'Arménie Magazine (un article et 38 commentaires du 19 octobre 2013 au 4 février 2016) et Horizon Weekly (un article et 7 commentaires du 21 octobre 2013 au 6 octobre 2014).

4- Une série de deux procès-verbaux du Comité exécutif et du Conseil municipal de la ville de Laval en lien avec l'édification du monument commémoratif aux victimes du génocide des Arméniens et trois articles de journaux locaux et régionaux.

Notre article est divisé en trois parties. Tout d'abord, il s'agit de faire un bref rappel de l'histoire et de la place de la communauté arménienne au Canada et au Québec. Ensuite, nous analyserons l'édification et l'inauguration du monument commémoratif aux victimes du génocide des Arméniens L'Espoir à Laval en nous référant aux débats qui ont marqué la construction du monument La Réparation à Montréal entre 1995-1998. Finalement, nous examinerons l'inscription et la représentation du discours mémoriel dans l'espace public

\footnotetext{
${ }^{6}$ CHABOt Joceline et GodIn Richard, "Histoire, mémoire et médias : connaissance et reconnaissance du génocide des Arméniens dans la presse québécoise, 1915-2005 ", in PÂQUET Martin (dir.), Faute et réparation au Canada et au Québec contemporains. Études historiques, Québec, Nota Bene, 2006, pp. 151-185. CHABOT, Joceline et THÉRIAULT Christine, "Une mémoire exemplaire ? La presse quotidienne québécoise et la demande de reconnaissance du génocide arménien (1965-1998) ", Questions de communication, série Actes 13, Dispositifs de médiation mémorielle, Nancy, 2011, pp. 231-149. CHABOT Joceline et GodIN Richard, "Temps, événements et mémoire médiatique : le cas du génocide des Arméniens dans la presse canadienne (1965-2005) ", Cahiers Mémoire et politique, no 2, Médias en jeu, enjeux de mémoire, 2014, pp. 113-128. 
médiatique à l'occasion du centenaire du génocide des Arméniens à la lumière des événements qui ont retenu l'attention et des thèmes qui ont été privilégiés par les médias québécois. II sera intéressant de comparer le récit médiatique en 2015 par rapport à celui que nous avons analysé pour les années 1965 à 2005 afin d'en mesurer les évolutions et les permanences ${ }^{8}$.

\section{Un brei rappel social et historique de la communauté arménienne au Canada et au Québec}

La communauté arménienne canadienne possède une histoire relativement ancienne puisque le premier émigrant arrivé au Québec s'installe dans la région minière de Thetford Mines en 1904\%. Il est le seul survivant de sa famille tuée lors des massacres de 1894-1896 commis dans l'Empire ottoman, sous le règne du sultan ABDUL-HAMID' ${ }^{10}$. Quelques années plus tard, suite au génocide de 1915, environ un millier de réfugiés, parmi lesquels on compte une centaine d'orphelins, s'installent en Ontario et au Québec". En 1930, on compte à Montréal 197 personnes d'origine arménienne. C'est à partir des années 1950 que le nombre d'Arméniens migrant au Canada augmente de façon significative. Ils proviennent de Grèce, d'Égypte et de Chypre. Le mouvement se poursuit dans les années 1960 et 1970 alors que les nouveaux arrivants viennent principalement du Liban et de la Syrie. Dans les années 1990, une nouvelle vague d'immigrants arméniens arrivent d'Irak. En 2006, selon les données du recensement de Statistique Canada, la population d'origine arménienne établie dans l'agglomération montréalaise s'élève à 23235 personnes $^{12}$. Mais, selon les Églises arméniennes de Montréal la communauté compterait plutôt 35000

\footnotetext{
${ }^{8}$ Nous avons procédé à l'analyse de corpus de presse similaires dans les articles cités à la note 6.

${ }^{9}$ Comme l'a montré Julien BAUER dans ses travaux sur l'histoire des minorités ethniques au Québec, la question des origines est importante. À ce titre, la recherche du "grand ancêtre ", c'est-à-dire le premier du groupe à avoir émigré, participe de la quête identitaire des immigrants, y compris de celle des Québécois dit "de souche " francophones et anglophones, BAUER Julien, Les minorités au Québec, Montréal, Boréal, 1994, p. 33

${ }^{10}$ Sur les massacres hamidiens, on peut lire DADRIAN Vahkan, Histoire du génocide arménien, Paris, Stock, 1996, chapitre 8, pp. 206-291. Aussi, le témoignage du consul français à Trébizonde, CILLIÈRE Alphonse, 1895 Massacres d'Arméniens, Toulouse, Privat, 2010, $281 \mathrm{p}$.

"KAPRIELIAN-ChuRChill Isabelle, "Armenian Refugees and their Entry into Canada, 1919-1930 ", Canadian Historical Review, 71, 1, 1990, pp. 87-93. SHIRINIAN Lorne, "Orphans of the Armenian Genocide with Special Reference to the Georgetown Boys and Girls in Canada ", in DeRmIRDJIAN Alexis (dir.), The Armenian Genocide Legacy, Londres, New York, 2016, pp. 44-66.

${ }^{12}$ Portrait statistique de la population d'origine ethnique arménienne, recensée au Québec en 2006. Immigration et Communautés culturelles. Gouvernement du Québec, 2010, 9 p.
} 
membres $^{13}$. Certains chercheurs estiment que ce dernier chiffre est beaucoup plus proche de la réalité ${ }^{14}$. La majorité de la communauté fait partie de la population immigrée, donc née à l'étranger (62,2\%), alors que près de $38 \%$ sont nés au Québec ${ }^{15}$.

Quoi qu'il en soit, une réalité indiscutable est le fait qu'en 2001, $98 \%$ de la communauté arménienne habite dans la grande région de Montréal, alors que pour l'ensemble de la population immigrée au Québec ce pourcentage se situe autour de $88 \%$. Cela étant, au fil des ans, la répartition géographique de la population d'origine arménienne s'est modifiée. En effet, comme bien d'autres immigrants, les Arméniens ont quitté la ville de Montréal pour s'établir dans les banlieues et plus particulièrement à Laval sur la rive nord de Montréal. En 2001, 60,2\% d'entre eux vivaient à Montréal et 31,2\% à Laval. En 2006, la situation avait encore évolué puisque $53,6 \%$ résidaient à Montréal et $32,9 \%$ à Laval ${ }^{16}$. Ainsi, selon les statistiques officielles, en 2011 , près de 8000 personnes de la communauté arménienne habitaient Laval, soit une augmentation de $18,5 \%$ par rapport aux données recueillies en $2006^{17}$.

Depuis la Seconde Guerre mondiale, la communauté arménienne s'est développée et s'est structurée autour d'organismes et d'institutions phares. Parmi ceux-ci, on retrouve les Églises de différentes confessions et les partis politiques. Mais on retrouve également plusieurs écoles, des associations sportives et culturelles plus ou moins liées aux Églises et aux partis. Actuellement, on compte plusieurs dizaines d'organismes et d'associations ainsi que des journaux témoignant de la vitalité de cette communauté ${ }^{18}$. D'ailleurs, la communauté arménienne est bien intégrée dans sa société d'accueil, maîtrisant le français et l'anglais, tout en étant plus scolarisée que l'ensemble de la population québécoise ${ }^{19}$.

\footnotetext{
${ }^{13}$ L'écart entre ces chiffres peut s'expliquer par le fait que certains membres de la communauté arménienne sont recensés à partir de leur lieu d'origine à savoir la Grèce, le Liban, etc. Il peut aussi relever d'une surestimation de la population d'origine arménienne par les représentants de la communauté afin d'augmenter leur importance en tant que groupe.

${ }^{14}$ LENOIR-ACHDJIAN Annick, Appréhender la nation, vivre en diaspora: regards arméniens, Thèse de doctorat en anthropologie, Université de Montréal, 2001, pp. 128-129.

${ }^{15}$ Portrait statistique de la population d'origine ethnique arménienne, op. cit.

${ }^{16}$ Portrait statistique de la population d'origine ethnique arménienne recensée au Québec en 2001. Immigration et Communautés culturelles. Gouvernement du Québec, 2015, 9 p. Portrait statistique de la population d'origine ethnique arménienne recensée au Québec en 2006, op. cit.

${ }^{17}$ Courrier de Laval, 16 mai 2015.

${ }^{18}$ LeNOIR-ACHDJIAN Annick, Appréhender la nation, op. cit., p. 138-140.

${ }^{19} 26,1 \%$ des Arméniens possèdent un diplôme universitaire contre 16,5\% pour l'ensemble de la population québécoise. Portrait statistique de la population d'origine ethnique arménienne recensée au Québec en 
Si la communauté ne constitue pas un groupe homogène, elle partage largement un même objectif, celui de maintenir et de promouvoir la culture et l'identité arménienne même si cette identité en diaspora est l'objet d'une renégociation en fonction du contexte social et culturel de la société d'accueil ${ }^{20}$. Les travaux des chercheurs qui se sont intéressés à la question identitaire montrent que la transmission de la culture arménienne passe par celle de son histoire et de sa mémoire. Au cœur de cette histoire et de cette mémoire, on retrouve l'événement traumatique qu'est le génocide de $1915^{21}$. Pour la majorité des Arméniens de la diaspora, il est clair que la Turquie doit reconnaître et réparer les torts subis lors du génocide.

Au Québec comme ailleurs en Occident, cette demande de reconnaissance du génocide arménien émerge au milieu des années 1960 avec la commémoration du 50e anniversaire des événements de $1915^{22}$. Mais c'est dans les années 1970 et surtout 1980, alors que la communauté arménienne est en plein essor, que cette demande s'accentue. Durant cette période, le 24 avril de chaque année ${ }^{23}$, la communauté commémore le crime resté impuni en organisant des manifestations. Cependant, il faut attendre les années 1990 avant que la communauté arménienne, bien intégrée dans la société d'accueil, formule de nouvelles revendications qui s'adressent non seulement aux représentants des autorités turques mais aussi aux autorités québécoises et canadiennes. Précisons qu'à compter de 1980, le gouvernement du Québec reconnaît le génocide des Arméniens. Dès lors, les représentants de la communauté arménienne cherchent à faire inscrire dans le calendrier civique montréalais et québécois le 24 avril journée commémorative du génocide arménien. Suite à l'adoption de lois, c'est chose faite en avril 1999 au conseil municipal de Montréal, et en décembre 2003, à l'Assemblée nationale du Québec.

Au niveau du gouvernement canadien, c'est le 21 avril 2004 que, suite à une motion d'une députée québécoise, la chambre des communes reconnaît le caractère génocidaire des massacres de 1915. Finalement, soulignons que le 7 avril 2015, la Conseil municipal de la ville de Laval reconnaît formellement le génocide de 1915 tout en soulignant depuis plusieurs années l'apport et le dynamisme de la

\footnotetext{
${ }^{20}$ KaSParian Sylvia, "Langues et identités des Arméniens en diaspora : État des lieux ", Revue Hommes et Migrations, dossier Diaspora arménienne et territorialité, 1265, 2007, p. 178.

${ }^{21}$ Hovanessian Martine, Le lien communautaire. Trois générations d'Arméniens, Paris, Armand Colin, 1992.

${ }^{22}$ ATTARIAN Varoujan, "La mémoire en diaspora. Actions pour la reconnaissance du génocide des Arméniens », in L'Actualité du génocide des Arméniens, Paris, Édipol, 1999, pp. 263-265. Снавот Joceline, et GODIN Richard, "Histoire, mémoire et médias », op. cit.

${ }^{23}$ Le 24 avril commémore la grande rafle de Constantinople de 1915 contre l'élite de la communauté arménienne qui marque le début du génocide.
} 
communauté lavalloise d'origine arménienne. Ainsi, depuis les années 2000, la reconnaissance du génocide des Arméniens par les autorités politiques à tous les niveaux de gouvernements est chose acquise au Québec et au Canada. La commémoration de cet événement est inscrite dans les calendriers civiques municipaux et dans celui de la province, alors que les citoyens du Québec sont appelés annuellement à exprimer leur solidarité avec la communauté arménienne à la mémoire des victimes du génocide de 1915.

\section{Un premier monument commémorant le génocide des Arméniens : L'Espoir}

Le 22 avril 1995, au parc public MARCELIN-WILSON, le maire de Montréal, Pierre BOURQUE, nouvellement élu, procède symboliquement à la première pelletée de terre et annonce l'érection prochaine d'un monument commémorant le génocide arménien. Or, quelques mois plus tard, en janvier 1996, le conseil municipal décide que le projet du monument commémoratif est reporté sine die. C'est ainsi que commence la saga du monument d'art public La Réparation. Au cours des mois suivants, les Montréalais apprendront que des membres de la communauté et des représentants consulaires turcs auraient fait pression pour que la Ville abandonne le projet de monument commémoratif. La polémique est lancée et les protestations s'intensifient alors que des représentants de la communauté arménienne mais aussi des groupes de la société civile québécoise dénoncent le recul de l'administration municipale dans ce dossier.

Après des mois de controverses et de débats qui engagent de nombreux acteurs sur la scène politique et médiatique, une solution est trouvée lorsque la Ville de Montréal annonce que le monument qui sera construit dans le parc public MARCELLIN-WILSON sera dédié à toutes les victimes de génocide. Selon les autorités municipales: le projet a été "globalisé » de manière à ce que le monument ne renvoie pas à un groupe ethnique en particulier ${ }^{24}$. Toutefois, une plaque placée à l'entrée du parc rappellera le génocide des Arméniens de 1915. En novembre 1998, La Réparation est enfin inaugurée mais seulement après que le monument ait été requalifié : désormais il doit commémorer toutes les victimes de génocides au $x x^{e}$ siècle - Hutus, Tutsis, Tsiganes, Juifs, Cambodgiens, Ukrainiens et tous ceux qui sont oubliés, comme il est mentionné sur le monument - amalgamant des crimes et des événements qui sont sans lien entre eux au risque d'une bana- 
lisation du crime de génocide. À terme, il fallait conclure à la difficile gestion des mémoires concurrentes et antinomiques - turque et arménienne - dans l'espace public de la société d'accueil.

Douze ans plus tard, en avril 2010, la Ville de Laval annonce que, suite à un accord de principe avec le Comité conjoint lavallois du Monument du génocide arménien (CCLMGA ${ }^{25}$ ), elle autorise l'aménagement d'un terrain pour l'édification d'un monument commémoratif du génocide des Arméniens qui rappellera d'autres crimes contre l'humanité ${ }^{26}$. Le monument doit être installé sur un terrain triangulaire situé au sud d'une autoroute (autoroute 440) et du côté ouest d'un grand boulevard (boulevard DANIEL-JOHNSON). À cette date, le concept du monument n'est pas encore arrêté et la Ville est toujours en discussion avec les membres du Comité. Toutefois, le Comité va rapidement procéder à l'élaboration d'un cahier des charges et à la demande de soumissions de projets de monument sur invitation. II va aussi mettre en ligne un site internet afin de recueillir des fonds pour en financer le coût.

Le 24 octobre 2012, le Comité exécutif de la ville de Laval, adopte à l'unanimité le choix de l'œuvre de l'artiste d'origine arménienne Arto TCHAKMAKCHIAN intitulé L'Espoir ${ }^{27}$. À cette date, il semble que le monument sera consacré à la commémoration du seul génocide des Arméniens. La sculpture de bronze qui mesure trois mètres et pèse une tonne est inaugurée le 20 octobre 2013 en présence des élus municipaux, de l'ambassadeur d'Arménie au Canada, Armen YEGANIAN, et des représentants de la communauté arménienne ${ }^{28}$. Selon Arto TCHAKMAKCHIAN, son œuvre renvoie à une fusion entre la crucifixion et la résurrection alors que l'intérieur évoque une mère en deuil abattue par la douleur mais qui se relève pour crier et prier, devenant ainsi porteuse d'espoir ${ }^{29}$.

Le monument commémoratif lavallois n'a pas suscité la polémique et la controverse comme l'avait fait le monument La Réparation dixhuit ans plus tôt et, dans cette perspective, il n'a pas fait l'objet d'articles dans les journaux nationaux consultés. S'il y a eu une requalification du monument entre l'annonce du projet en 2010 et sa mise en

\footnotetext{
${ }^{25}$ Ce comité regroupe des représentants de quatre Églises arméniennes établies à Montréal et à Laval.

${ }^{26}$ FORTIN Geneviève, "Un monument à la mémoire du génocide arménien », Le Courrier de Laval, 13 avril 2010.

${ }^{27}$ Procès-verbal d'une séance du Comité exécutif de la ville de Laval, 24 octobre 2012. Province de Québec, Ville de Laval. Vol. 774 CE-2012/6376, p. 42.

${ }^{28}$ "Genocide Monument in Canada inaugurated », Horizon Weekly, 21 octobre 2013.

${ }^{29}$ "Accueil Forums, Nouveau monument commémoratif», Nouvelles d'Arménie magazine, 19 octobre 2013. 
œuvre en 2012, c'est pour affirmer sa vocation de commémorer spécifiquement le génocide des Arméniens et non pas tous les génocides du $x x^{e}$ siècle. D'ailleurs, le journal Horizon Weekly n'hésite à affirmer qu'il s'agit du premier et du seul monument commémorant exclusivement cet événement dans l'espace public canadien ${ }^{30}$.

Une autre différence par rapport au monument La Réparation tient à la symbolique religieuse fortement marquée dans la description qu'en livre son auteur. La Réparation, œuvre de l'artiste québécoise Francine LARIVÉE, faisait appel à une symbolique plus largement culturelle rappelant la fragilité et la destruction de la culture arménienne symbolisée par « [...] la maison brisée (marbre blanc), ouverte en son milieu par le coup du massacre [...]. L'absence de fondation durable exprime la précarité de l'enracinement nouveau, corollaire de la perte du lieu, du territoire, du pays $[. . .]^{31}$ ". Le caractère esthétique de l'œuvre de TCHAKMAKCHIAN et sa symbolique sont assez différentes du monument montréalais.

Finalement, il faut aussi signaler que l'œuvre ne s'inscrit pas à proprement parler dans le tissu social et géographique de la communauté arménienne lavalloise, contrairement à La Réparation qui a été édifiée dans un parc public situé au cœur du quartier où l'on retrouve la plus forte concentration de membres de la communauté arménienne à Montréal. En effet, L'Espoir semble abandonné au bord de l'autoroute 440 , à proximité d'un centre commercial sans lien direct avec les quartiers de résidence de la communauté arménienne.

C'est d'ailleurs cet emplacement excentré et le choix artistique du monument qui vont susciter les critiques les plus nombreuses au sein du grand public et plus particulièrement chez les membres de la communauté arménienne. En effet, contrairement à La Réparation, ce ne sont pas ses visées mémorielles non plus que le rappel des événements historiques qui retiennent l'attention de ses détracteurs mais plutôt les qualités esthétiques du monument. Ainsi, sur le forum web armenews.com ${ }^{32}$, plusieurs personnes considèrent que l'œuvre rate sa cible parce qu'elle ne réussit pas à rendre lisible le génocide ou encore parce qu'elle n'entretient aucun lien avec la culture arménienne. Si d'aucuns estiment qu'au contraire, le monument incarne justement

\footnotetext{
30 "Genocide Monument in Canada ".

31 Ville de Montréal, 1998, Contrat d'exécution d'œuvre d'art. Description de l'œuvre par Francine LARIVÉE, document annexe.

32 II s'agit du site web du magazine Nouvelles d'Arménie qui, sous la direction d'un modérateur, offre à ses lecteurs un lieu pour exprimer leur opinion par rapport aux dernières nouvelles concernant la communauté 
"le peuple arménien torturé33 ", d'autres sont d'avis qu'il représente bien "le côté pétrifié de notre mémoire sur l'horreur ${ }^{34}$ ". Certaines critiques, non sans humour, évoquent le caractère trop abstrait de l'œuvre qui ressemble à un tir bouchon alors qu'un lecteur dénonce le mauvais goût qui prévaut dans l'art public contemporain, comme c'est le cas, écrit-il depuis «le Centre POMPIDou, une usine à gaz à Paris $^{35}$ ", sans que l'on voit très bien le lien entre le monument à Laval et le musée à Paris. En somme, ce qui suscite la critique dans le cas du monument L'Espoir c'est le fait que l'œuvre ne semble pas rencontrer l'attente de son public au moins dans sa dimension expressive et formelle, même si elle a été commandée par les représentants de la communauté arménienne lavalloise et créée par un artiste d'origine arménienne $e^{36}$.

\section{Quand le témoin est saisi par les médias : une relation nouvelle entre témoin et médias?}

Dans cette dernière partie, nous voulons examiner plus spécifiquement les pratiques et le discours autour des événements commémorant le $100^{\mathrm{e}}$ anniversaire du génocide. Lors de nos travaux précédents, nous avons analysé la construction et l'inscription des enjeux entourant la mémoire du génocide des Arméniens dans l'espace public québécois entre 1965 et $2005^{37}$. Une chronologie des thématiques nous a permis d'affirmer qu'il n'y a pas de transformation radicale des représentations mémorielles dans le récit médiatique mais plutôt des moments forts qui correspondent à l'expression d'une mémoire communautaire partagée autour de la demande de reconnaissance du génocide arménien adressée d'abord et avant tout la Turquie (19651985) mais aussi, de plus en plus, à la société d'accueil québécoise et canadienne (1985-2004). Ces travaux qui s'articulaient autour des discours, des pratiques et des représentations mémoriels contribuaient à éclairer les relations sociales, politiques entre la communauté arménienne diasporique et la société d'accueil.

\footnotetext{
${ }^{33}$ Accueil Forums, Nouveau monument commémoratif, Nouvelles d'Arménie magazine, 21 octobre 2013.

34 Ibid.

35 Ibid.

${ }^{36}$ L'art contemporain et l'art public font souvent l'objet de critiques de la part des médias qui dénoncent le fait que les œuvres ne soient pas accessibles à un large public. Voy. à ce propos: ArBour Rose-Marie, " $\mathrm{A}$ quoi sert l'art contemporain? „, ETC, no 48, 1999-2000, p. 6-10.

37 Voy. note 3 ci-dessus.
} 
En 2015, la communauté arménienne québécoise s'est mobilisée autour de la commémoration du centenaire du génocide. Cette mobilisation annuelle autour de la date anniversaire du 24 avril a évidemment été beaucoup plus importante et médiatisée à l'occasion du centenaire. Les médias québécois ont fait écho à ces manifestations et ont rendu compte des événements nationaux et internationaux autour de la commémoration du centenaire.

Une analyse des articles de notre corpus nous permet d'affirmer que les thèmes abordés demeurent sensiblement les mêmes à savoir : la mémoire des victimes, le déni turc du caractère génocidaire des événements, la demande de reconnaissance au niveau internationale et l'absence de réparation pour les victimes de 1915 et leurs descendants. Néanmoins, on note aussi une ré-articulation de ces thèmes dans la trame narrative médiatique. En effet, en cette année du centenaire, la demande de reconnaissance et le déni turc dominent l'actualité à travers un certain nombre d'événements largement médiatisés. Au premier chef, c'est la déclaration du pape François à la basilique Saint-Pierre-de-Rome, le 12 avril 2015 qui fait la manchette des médias. À cette occasion, les journaux rapportent l'expression utilisée par le Pape qualifiant les événements tragiques de 1915 de "premier génocide du $x x^{e}$ siècle $^{38}$ ". Du même souffle, la réaction des autorités turques surgit en contrepoint de la nouvelle: ainsi, les journaux rappellent les déclarations du ministère turc des Affaires étrangères dénonçant le "point de vue sélectif ${ }^{39}$ " du Pape et celle du président Erdogan qui assimile les propos du Souverain Pontife "à des délires ${ }^{40}$ ". Dans le même ordre d'idées, les journaux présentent aussi les dénonciations d'Ankara à la suite des déclarations du président russe, Vladimir POUTINE, du président fédéral allemand, Joachim GAUCK, et du président français, François HoLLANDE, sur le génocide de 1915 : ERDOGAN les accuse "de soutenir les revendications basées sur les mensonges arméniens ${ }^{41}$ ".

Dans les médias, c'est l'occasion de rappeler le déni turc et ses arguments: d'abord, les Arméniens ont été victimes d'une guerre civile et la majorité sont morts de faim et de maladie. Ensuite, le nombre des morts est aussi important du côté turc que du côté arménien. Finalement, la guerre des chiffes est toujours très présente dans les articles recensés, au chiffre d'un million et demi d'Arméniens exter-

\footnotetext{
38 "Le Pape François évoque le génocide arménien », Le Devoir, 13 avril 2015.

39 Ibid.

40 La Presse, 14 avril 2015.

${ }^{41}$ La Presse, 24 avril 2015.
} 
minés, la Turquie oppose le nombre de 300000 à 600000 victimes ${ }^{42}$. Sur le thème de la reconnaissance, les médias font une large place au décompte des États qui reconnaissent le génocide des Arméniens, en rappelant que depuis 2004 le Canada est au nombre de ceux-ci. Ils rapportent aussi les résistances et les hésitations de certains gouvernements à s'engager sur la voix de la reconnaissance, notamment celui des États-Unis ${ }^{43}$. Les journaux citent la déclaration du secrétaire général de la Maison Blanche, Dennis McDonough, qui parle d'une "période horrible " qui a fait 1,5 million de morts mais en évitant soigneusement d'utiliser le terme de génocide ${ }^{44}$.

L'autre événement très médiatisé est la marche pour commémorer le génocide qui s'est déroulée le 3 mai 2015 à Montréal. Cette manifestation annuelle a revêtu un caractère particulier en 2015 puisque le Comité canadien du centenaire du génocide qui en était le principal organisateur avait invité les représentants et les membres des communautés victimes de génocides et de crimes de masse à venir dénoncer tous les génocides. C'est ainsi que le président du comité, Mher KaRAKASHIAN, évoque l'événement:

En cette année du centenaire, la communauté a une nouvelle vocation; celle de non seulement se souvenir des victimes de tous les génocides du $\mathrm{XX}$ e siècle, mais aussi de sensibiliser la population pour éviter que d'autres atrocités de la même ampleur soient répétées ${ }^{45}$.

Si les thèmes traités dans le cadre de la nouvelle sont sensiblement les mêmes que ceux que nous avions repérés dans nos analyses précédentes, l'analyse des articles du corpus révèle une dynamique testimoniale qui rend compte d'une nouvelle relation entre le journaliste et le témoin. D'emblée, il faut souligner que l'accentuation du discours testimonial dans les médias, y compris dans les journaux, n'est pas une nouveauté. Comme l'a montré Jean-François TÊTu, la mise en scène de l'émotion et notamment du spectacle de la souffrance et de l'injustice

\footnotetext{
${ }^{42}$ Sur la politique négationniste, BaYrakTAR Seyhan, "The Politics of Denial and Recognition : Turkey, Armenian and the EU », in DeRMIRDJIAN Alexis (dir.), The Armenian Genocide, op. cit., pp. 197-211. Sur la mécanique négationniste, HOVANNISIAN Richard G., " L'hydre à quatre têtes du négationnisme. Négation, rationalisation, relativisation, banalisation ", in L'Actualité du génocide des Arméniens, Paris, Édipol, 1999, pp. 143-175. 43 Pour une mise au point sur la question de la reconnaissance du génocide des Arméniens par les autorités politiques américaines et sur le rôle des médias américains dans le traitement de cette question : ZARIFIAN Julien, "Les médias américains et la (non)-reconnaissance du génocide des Arméniens aux États-Unis ", Revue française d'études américaines, n 142, 2015/1, pp. 55-71. Contrairement aux médias québécois que nous avons analysés dans cette étude, certains médias américains - par exemple, le Washington Times - refusent encore d'utiliser le terme de génocide pour parler du cas arménien et accueillent sur leurs plateaux et dans leurs pages des auteurs négationnistes. ZARIFIAN Julien, « Les médias américains, op. cit., p. 56.

${ }^{44}$ "Massacres d'Arméniens : la Maison-Blanche évite le mot génocide », La Presse, 21 avril 2015.

45 "Une marche en mémoire des génocides », Metro, 3 mai 2015.
} 
à travers le témoignage des victimes est depuis longtemps présente dans le discours médiatique ${ }^{46}$. Ce qui semble marquer un changement dans le dispositif testimonial tel qu'il se dévoile dans le corpus analysé, c'est la place de plus en plus grande du témoin dans le récit médiatique. En effet, le journaliste ne fait plus seulement advenir la parole du témoin, agissant ainsi comme un médiateur entre celui-ci et le lecteur, mais désormais le témoin devient une source et une ressource pour le journaliste. Le témoin accompagne le journaliste dans sa quête documentaire sur le génocide et il livre un récit chargé d'émotion des conséquences dramatiques de ces événements sur son parcours et sur celui de sa famille. Ultimement, le journaliste devient lui-même témoin et militant.

Tous les journaux (locaux, régionaux nationaux) consultés sont à la recherche du témoin qui livrera son expérience personnelle des conséquences du génocide de 1915 sur sa famille. Est mis en avant la mémoire et la parole du témoin de $2^{\mathrm{e}}$, voire de $3^{\mathrm{e}}$ génération, c'est-àdire non pas celui qui a vu et vécu le génocide (aujourd'hui les témoins directs capables de raconter leur histoire sont morts) mais celui qui assure la transmission de la mémoire des victimes ${ }^{47}$. Ainsi, le journaliste du Devoir, Jean-Frédéric LÉGARÉ-TREMBLAY, interroge un descendant du génocide établi à Montréal, Jean MEguerditchian, qui lui raconte l'histoire de son père. Celui-ci a témoigné de son parcours dans un texte d'une quarantaine de pages. À partir de ce récit, Jean retrace le parcours de Garabed, son père, né à Zeitoun, entremêlant le récit familial et l'explication historique de l'extermination de la population arménienne. II raconte le récit tragique de Garabed à compter de sa

\footnotetext{
${ }^{46}$ TÊTU Jean-François, "L'émotion dans les médias : dispositifs, formes et figures ", Mots. Les langages du politique, no 75, 2004, p. 9. Aussi, sur cette question: FLEURY Béatrice, WALTER Jacques, «La construction de la figure d'Esther Fogiel au procès PAPON : une écriture entre l'histoire et le récit de l'intime ", Génocide: les figures de la victime, La Pensée et les Hommes, n 85, 2012, pp. 117-133.

47 Sur la question de la mémoire des génocides et des défis de sa transmission intergénérationnelle les travaux sont très nombreux, nous retiendrons: ALTOUNIAN Janine, L'intraduisible. Deuil, mémoire, transmission, Paris, Dunod, 2005, 206 p. Fass Paula S., Inheriting the Holocaust : A Second Generation Memoir, New Brunswick, N.J., Rutgers University Press, 2011, 193 p. HIRSH Mariane, The Generation of Postmemory. Writing and Visual Culture after the Holocaust, New York, Columbia University Press, 2012, plus particulièrement le chapitre 1 «Familial Postmemories and Beyond, pp. 29-102. PICKERING Michael et KEIGHTLEY Emily, "Communities of Memory and the Problem of Transmission ", European Journal of Cultural Studies, 16, 1, 2012, pp. 115-131. TAHER Saaz, "Comparaison des imaginaires nationaux. Les deuxième et troisième générations d'Arméniens à Montréal ", in CHABOt Joceline, Doucet Marie-Michèle, KaSPARIAN Sylvia, THIBAULT Jean-François (dir.), Le Génocide des Arméniens. Représentations, traces, mémoires, Québec, Paris, Presses de l'Université Laval, Éditions Hermann, 2017, pp. 171-185.
} 
déportation, puis de son refuge à Bagdad où il sera éduqué par les frères trappistes en français, jusqu'à sa venue à Montréal en 1972. De sa famille, seuls deux cousins ont survécu ${ }^{48}$.

Dans le journal local L'Écho de Laval, Fanny ARNAUD donne la parole à une descendante de la troisième génération, Garine OHANIAN, qui raconte l'histoire de la famille de son grand-père Manoug KATCHADOURIAN, tout en retraçant les principaux événements qui ont frappé les civils arméniens à compter du 24 avril 1915. Contrairement à la majorité de sa famille, son grand-père a survécu à la déportation parce qu'il a été adopté par une femme kurde. II a alors renoncé à sa langue et à sa religion. Au terme d'une vie très difficile, il est venu s'installer avec sa famille à Laval en 1977. Sa petite-fille conclut ainsi son récit : "Nous ressentons tous la douleur de nos ancêtres " ${ }^{49}$.

Dans un autre article, Le Devoir livre directement le témoignage de Luc Vartan BARONIAN qui retrace le parcours de sa famille, en commençant par son grand-père, rescapé du génocide et réfugié en Égypte, qui ne parlait jamais de ces événements, jusqu'à son père arrivé à Montréal en 1965. Son père entreprendra de connaître les détails de I'histoire familiale à la lumière de l'extermination des Arméniens. II apprendra que ses grands-parents ont été égorgés et que seuls ont survécu à la déportation deux cousins et une cousine qui a dû abandonner son enfant à une famille de Bédouins. Ici, BARONIAN introduit le récit tragique de cette femme et de son enfant qu'elle retrouve des années plus tard: "Marié, ce dernier [son enfant] ne voulut pas repartir [avec sa mère], mais, dans un geste tragique qui suscite néanmoins l'espoir, lui remet son plus jeune fils ${ }^{50}$ ".

Nous pourrions multiplier ainsi les témoignages tirés de notre corpus, nous terminerons avec un dernier exemple, celui du journaliste-témoin. II s'agit du cas de Rima ElkouRI, chroniqueur à La Presse, qui, dans un dossier consacré au centenaire du génocide, part à la recherche de ses ancêtres arméniens ${ }^{51}$. Elle se rend ainsi pour la première fois en Turquie, à Mardin, sur les traces de la famille de son grand-père Naïm Karazivan. Comme elle le rappelle, il s'agit pour elle d'un voyage dans le "cimetière de la mémoire de mes ancêtres ${ }^{52}$ ". Elle ne connaît que des brides de leur parcours à compter de leur

\footnotetext{
${ }^{48}$ LÉGARÉ-Tremblay Jean-Frédéric, “Cent ans de mémoire arménienne », Le Devoir, 22 avril 2015.

49 ARNAUD Fanny, " Génocide arménien : le témoignage d'une famille de Laval ", L'Écho de Laval, 4 mai 2015.

50 BARONIAN Luc Vartan, "De l'Arménie du génocide à la Syrie dévastée », Le Devoir, 20 avril 2015.

${ }^{51}$ ElkourI Rima, "Sur les pas de mon grand-père "La Presse, 4 avril 2015; "Cent ans de tristesse ", La Presse, 6 avril 2015 ; "Survivre à I'horreur ", La Presse, 6 avril 2015; "Le grand tabou des Arméniens islamisés ", La Presse, 7 avril 2015.

${ }^{52}$ ElkourI Rima, "Sur le pas de mon grand-père »,op. cit.
} 
déportation de Mardin. Sur place, à Mardin, elle interroge sans grand succès des personnes susceptibles de lui fournir des informations sur sa famille. Afin de mieux comprendre son parcours familial et de le situer plus largement dans l'histoire des Arméniens de Mardin, elle procède à l'interview de l'historien Yves TERNON ${ }^{53}$, car, précise-t-elle, ni son arrière-grand-mère, ni son grand-père n'ont parlé des événements qu'ils ont vécus à leurs enfants. La construction narrative de ses articles mêle habilement récit familial et explication historique sur le génocide. À travers ses textes, elle adopte une posture éthique et politique puisqu'elle dénonce le silence et le déni turc et met en avant les revendications de la communauté arménienne.

\section{Conclusion}

Dans le cadre de cet article, nous avons interrogé les pratiques et le discours sur le génocide des Arméniens dans l'espace public médiatique québécois en tenant compte de l'histoire de la communauté arménienne québécoise et de l'évolution des politiques mémorielles québécoise et canadienne sur cette question. À travers deux séries d'événements, la construction d'un monument commémoratif à Laval et les manifestations entourant le centenaire du génocide des Arméniens en 2015, nous avons analysé les continuités et les changements dans le récit médiatique du génocide arménien. En commençant par le monument commémoratif L'Espoir érigé à Laval, là où depuis une dizaine d'années a migré une partie de la communauté arménienne québécoise. Notre analyse a montré que l'érection de ce monument n'a pas suscité de débat dans l'espace médiatique contrairement au monument La Réparation construit à Montréal dans les années 1990. Si d'aucuns ont critiqué les qualités esthétiques de l'œuvre, aucune controverse politique et mémorielle n'a été répercutée publiquement dans les médias. II semble que la reconnaissance du génocide des Arméniens par les gouvernements québécois et canadien ait contribué à apaiser le débat autour de cette question.

L'analyse des articles portant sur le deuxième événement médiatique soit la commémoration du centenaire du génocide a montré une certaine évolution du récit médiatique. Les thèmes les plus souvent abordés sont ceux de la demande de reconnaissance du génocide et le déni turc. Dans ce cadre, les médias ont marqué les avancées au niveau international - déclaration du pape François, discours des

${ }^{53}$ Rappelons que dans le cadre de ses recherches Yves TERNON s'est intéressé tout particulièrement au cas des Arméniens de la région de Mardin : TERNON Yves, "Mardin 1915 », Revue d'histoire arménienne contemporaine, no spécial 76b, juin 2002, 400 p.. 
chefs politiques russe, allemand et français - et la permanence des arguments d'Ankara présentés à travers les discours offusqués des autorités turques et plus particulièrement du président ERDOGAN. Par ailleurs, à travers la manifestation du 3 mai 2015 à Montréal, les médias ont souligné l'ouverture de la communauté à la commémoration de toutes les victimes de crimes de masse mais aussi la transmission de la mémoire de ce génocide aux jeunes générations et à l'ensemble de la société d'accueil.

Dans cette perspective, le changement majeur noté à l'analyse de notre corpus est dans la relation dialogique entre le journaliste et le témoin. Ce couple n'est évidemment pas récent dans les médias mais ses rapports ont évolué : si le journaliste organise par sa médiation le dispositif mémoriel issu de la parole du témoin, on constate qu'une place de plus en plus grande est laissée au discours direct du témoin. Un discours chargé d'émotion qui dénonce l'injustice, c'est-à-dire le déni et l'absence de réparation. C'est à travers le prisme du récit de vie du témoin de $2^{\mathrm{e}}$ et de $3^{\mathrm{e}}$ générations que l'ensemble des médias analysés présentent et représentent l'histoire et la mémoire du génocide des Arméniens. Comme l'a noté Annette WIEVIORKA, le témoin est non seulement au cœur de son histoire mais il devient lui-même l'Histoire ${ }^{54}$. Ultimement, le journaliste peut aussi devenir témoin et militant: son témoignage contribue à rendre vivant le récit historique, mais il lui offre aussi l'occasion d'une réparation symbolique pour le crime impuni en faisant entendre les revendications de sa communauté à l'ensemble des citoyens. 